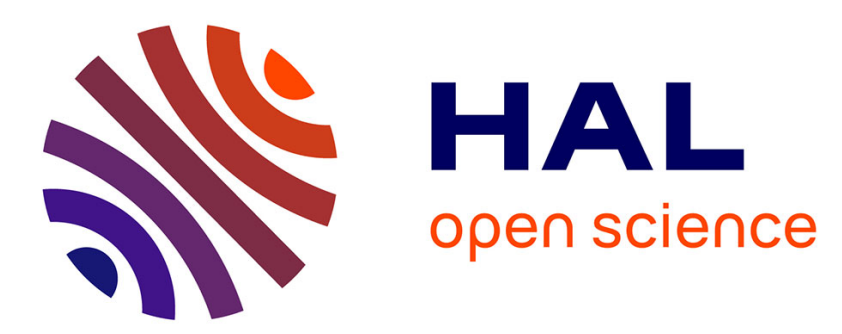

\title{
Anisotropie du trainage de fluctuations thermiques dans le domaine de Rayleigh
}

R. Vergne, J.L. Porteseil, Z. Blazek

\section{To cite this version:}

R. Vergne, J.L. Porteseil, Z. Blazek. Anisotropie du trainage de fluctuations thermiques dans le domaine de Rayleigh. Revue de Physique Appliquée, 1977, 12 (7), pp.979-987. 10.1051/rphysap:01977001207097900 . jpa-00244280

\section{HAL Id: jpa-00244280 https://hal.science/jpa-00244280}

Submitted on 1 Jan 1977

HAL is a multi-disciplinary open access archive for the deposit and dissemination of scientific research documents, whether they are published or not. The documents may come from teaching and research institutions in France or abroad, or from public or private research centers.
L'archive ouverte pluridisciplinaire HAL, est destinée au dépôt et à la diffusion de documents scientifiques de niveau recherche, publiés ou non, émanant des établissements d'enseignement et de recherche français ou étrangers, des laboratoires publics ou privés. 


\title{
REVUE DE PHYSIQUE APPLIQUÉE
}

\author{
Classification \\ Physics Abstracts \\ 8.550
}

\section{ANISOTROPIE DU TRAINAGE DE FLUCTUATIONS THERMIQUES DANS LE DOMAINE DE RAYLEIGH}

\author{
R. VERGNE, J. L. PORTESEIL et Z. BLAŽEK (*) \\ Laboratoire de Magnétisme du C. N. R. S. 166 X 38042 Grenoble Cedex, France
}

(Reçu le 15 décembre 1976, accepté le 4 avril 1977)

\begin{abstract}
Résumé. - Dans un échantillon polycristallin ferromagnétique dont la structure en domaines élémentaires est anisotrope, la valeur de la susceptibilité différentielle irréversible dépend de la direction dans laquelle on la mesure. Ce fait entraîne une variation angulaire du traînage de fluctuations thermiques qui lui est proportionnel. Mais ce n'est pas là la seule cause d'anisotropie de ce traînage car le champ de fluctuations lui-même peut dépendre de la configuration en domaines. Nous analysons cet effet en nous bornant à un problème plan dans le cas où on a obtenu une structure en domaines anisotrope par une désaimantation alternative d'un échantillon polycristallin ayant plusieurs axes de facile aimantation. Pour que la mesure ait un sens, il faut conserver la répartition angulaire des polarisations caractérisant la structure en domaines initiale. Cette restriction impose d'appliquer des champs assez faibles devant $H_{\mathrm{c}}$; c'est-à-dire de travailler dans le domaine de Rayleigh. L'expérience confirme l'existence de l'effet prévu. Les résultats s'interprètent à partir du modèle d'anisotropie de la désaimantation en supposant le champ de traînage isotrope, ce qui permet de conclure que l'essentiel du phénomène est dû à la variation de la susceptibilité différentielle irréversible. Cependant, un examen plus serré des résultats expérimentaux laisse prévoir une légère anisotropie du champ de fluctuations.
\end{abstract}

Abstract. - If the domain structure of a polycrystalline ferromagnet is anisotropic, the irreversible susceptibility $S_{\mathrm{i}}$ depends on the direction along which it is measured. As a consequence, the magnetic thermofluctuation after-effect, which is proportional to $S_{\mathrm{i}}$, is expected to exhibit an angular dependence. This is not the only reason why the magnetic after-effect should be anisotropic, as the thermofluctuation field itself may depend on the statistical features of the domain structure. We analyze these effets in the case of the anisotropic domain structure created in a polycrystal by an alternating demagnetization. In order to preserve this initial domain structure, the applied fields should be much smaller than $H_{\mathrm{C}}$ (Rayleigh region). One experimentally finds the expected effect. The results are well interpreted by assuming that the thermofluctuation field is isotropic, which shows that the observed phenomena are mainly due to the angular dependence of $S_{\mathrm{i}}$. However, a closer examination of the experimental results suggests that the thermofluctuation field may be slightly anisotropic.

1. Introduction. - L'expérience montre [1] qu'une substance magnétique, soumise à un champ constant $H_{0}$, prend une aimantation $J$ qui est la somme de deux termes, l'un constant, l'autre évoluant au cours du temps.

$$
J=J_{0}+J_{1}(t) .
$$

Nous supposerons ici que le temps $t$ écoulé depuis l'application du champ $H_{0}$ est suffisant pour que soient éteints les courants induits. L. Néel [2] a alors montré que l'évolution de l'aimantation au cours du temps peut être due à deux mécanismes différents : la diffusion de défauts de réseau ponctuels dans le cristal ferromagnétique d'une part et l'agitation thermique de l'aimantation spontanée dans les domaines de

(*) National Research Institut for Materials, S. V. U. M. Prague I, Opletalova 25, Czechoslovakia.
Weiss d'autre part. Nous nous intéresserons uniquement ici au traînage de fluctuations thermiques. C'est un phénomène très général qui présente un caractère irréversible contrairement au traînage de diffusion qui est réversible du point de vue magnétique et ne se produit que dans des conditions bien déterminées.

Il est commode de supposer que les déplacements de paroi qui sont à l'origine de la variation d'aimantation $J_{1}(t)$ sont causés par un champ interne fictif $h(t)$ que Néel appelle champ de traînage. L'aimantation que l'on observe à l'instant $t$ est équivalente à celle que l'on observerait si le traînage n'existait pas et si la substance était soumise au champ effectif $H_{\mathrm{e}}$ fonction du temps :

$$
H_{\mathrm{e}}=H_{0}+h(t) .
$$

L'agitation thermique fait que la direction de l'aimantation spontanée ne reste pas rigoureusement 
uniforme à chaque instant à l'intérieur d'un domaine élémentaire. Il en résulte un champ de dispersion qui fluctue aléatoirement autour d'une valeur moyenne nulle à des fréquences très élevées. Ce champ s'ajoutant ou se retranchant au champ appliqué peut aider les parois à franchir les obstacles qui s'opposent à leur déplacement. Les variations d'aimantation ainsi acquises dépendent de la valeur moyenne des fluctuations maximales du champ dû à l'agitation thermique. L. Néel [3] a obtenu pour $h(t)$ une loi de la forme : $h(t)=S(Q+\log t) . S$ est homogène à un champ et est une constante du corps ferromagnétique étudié. Ce facteur est lié en particulier aux volumes des discontinuités de Barkhausen. Il croît avec la température absolue comme $T^{1 / 2} . Q$ est une constante sans dimension qui varie peu avec la substance étudiée et qui est de l'ordre de 40 à 50 . $t$ est le temps écoulé depuis l'application du champ magnétique. Pour que la relation donnant $h(t)$ soit applicable, il faut que le temps $t$ soit suffisant pour que la quantité $(Q+\log t)$ soit toujours positive. En un point de la courbe d'aimantation, $J_{0}, H_{0}$ où la susceptibilité différentielle irréversible est $S_{\mathrm{i}}$, le champ de traînage provoque l'apparition d'un terme d'aimantation irréversible de la forme :

$$
J_{1}(t)=S_{\mathrm{i}} S(Q+\log t) .
$$

C'est essentiellement, J. C. Barbier [4] qui, à partir de la théorie de Néel, a établi les principaux caractères du traînage de fluctuations thermiques. Il a montré, en particulier, que la quantité $S$ est sensiblement constante pour une substance donnée dans tout le domaine d'hystérésis et est de l'ordre de $H_{\mathrm{c}} / 1000$. L'expérience montre qu'elle croît comme $T^{3 / 4}$ et non comme $T^{1 / 2}$, comme le prévoyait la théorie de Néel.

1.1 ANISOTROPIE DU TRAINAGE DE FLUCtUAtions THERMIQUES. - La structure en domaines d'un échantillon polycristallin est en général très complexe. Il existe un grand nombre de parois dont les vecteurs polarisation $\left({ }^{1}\right)$ sont répartis suivant toutes les directions de l'espace. Quand un tel échantillon est maintenu sous champ constant, chaque paroi a la possibilité de se déplacer sous l'action du champ aléatoire local dû aux fluctuations thermiques et on observe le long d'une direction macroscopique de mesure, une variation d'aimantation qui est la projection suivant cette direction de l'ensemble des variations d'aimantation dues aux déplacements élémentaires des parois. La susceptibilité différentielle irréversible, suivant une direction de mesure, dépend de l'anisotropie de la configuration en domaines. De ce fait, on doit s'atten-

(1) Remarque : Le vecteur polarisation $\mathbf{P}$, relatif à une paroi de Bloch séparant deux domaines élémentaires aimantés respectivement suivant $\mathbf{J}_{1}$ et $\mathbf{J}_{2}$ est, par définition, le vecteur : $\mathbf{P}=\mathbf{J}_{2}-\mathbf{J}_{1}$. Le déplacement de la paroi affectant un volume $\Delta V$ entraîne une variation d'aimantation $\mathrm{P} \Delta V$ dans la direction de $\mathbf{P}$. dre à une variation angulaire du traînage qui reflète cette anisotropie.

Mais ce n'est pas la seule cause possible d'anisotropie. En effet, il est très vraisemblable que la répartition spatiale du champ de fluctuation est également liée à la répartition en domaines. En l'absence de théorie, nous aborderons la question de la manière suivante : nous supposerons d'abord que le champ de fluctuations est isotrope, nous calculerons la variation angulaire du traînage sous champ pour une configuration en domaines donnée et nous confronterons le résultat avec l'expérience. S'il s'avère qu'il existe des écarts systématiques, nous serons amenés à remettre en cause l'hypothèse d'isotropie du champ de fluctuations. Une démarche analogue est possible à partir de la mesure des susceptibilités différentielles irréversibles qui, dans certaines conditions, donnent accès à $h_{\mathrm{t}}$. Ce qui vient d'être dit ne préjuge en rien sur la cause de l'anisotropie de la structure en domaines.

Dans ce qui suit, nous allons envisager le cas où cette structure anisotrope a pour cause la désaimantation de l'échantillon. Rappelons brièvement ce qu'est le phénomène d'anisotropie de la désaimantation dont nous avons développé un modèle par ailleurs [5]. Pour obtenir un état désaimanté, c'est-àdire un état d'aimantation macroscopique nulle, on applique le plus souvent à l'échantillon un champ alternatif évanescent dont l'amplitude maximale est quelques fois le champ coercitif $\left(\mathfrak{H}_{\mathrm{m}} \geqslant 3 H_{\mathrm{c}}\right)$. Un tel processus conduit à un état désaimanté anisotrope si la substance possède plusieurs axes de facile aimantation. En effet, dans un cristallite, au cours de la désaimantation alternative, les directions de facile aimantation ne sont plus énergétiquement équivalentes. La direction la plus proche du champ de désaimantation est privilégiée et ceci d'autant plus que l'amplitude maximale $\mathscr{H}_{\mathrm{m}}$ du champ de désaimantation est plus grande. Au cours de la désaimantation, les vecteurs aimantation spontanée $\mathbf{J}_{\mathbf{S}}$ auront tendance à se placer d'abord suivant les directions de facile aimantation de plus basse énergie. Il en résulte une répartition anisotrope de ces vecteurs dans le polycristal désaimanté et une structure en domaines qui reflète cette anisotropie. Ce phénomène n'existe plus dans une substance uniaxiale, car au niveau du cristallite, il n'y a plus de choix possible, une seule direction étant privilégiée. Dans un polycristal réel, l'existence de défauts fait que dans certaines régions, les axes de facile aimantation ne sont plus énergétiquement équivalents. On a un caractère uniaxial local très prononcé, entraînant dans ces régions, une configuration en domaines qui ne dépend pas du mode de désaimantation. Si ces régions sont réparties au hasard dans le polycristal, il en résulte pour l'ensemble de celles-ci un état désaimanté isotrope par compensation. Dans un tel matériau, une partie seulement sera sensible au phénomène d'anisotropie de la désaimantation.

Nous avons fait une étude expérimentale de l'in- 
fluence d'un état désaimanté anisotrope sur l'aimantation dans le domaine de Rayleigh [6]. Celle-ci nous incite à attribuer l'origine du phénomène à une modification des centres de nucléation qui prennent naissance dans les régions perturbées du cristal, au cours de la désaimantation. Ce sont elles qui conditionnent la structure en domaines qui dépend du type de désaimantation.

L'étude de l'aspect directionnel du traînage de fluctuations thermiques dans le domaine de Rayleigh, c'est-à-dire pour des valeurs du champ qui ne modifient pas sensiblement la répartition angulaire des polarisations $\left({ }^{2}\right)$ caractérisant la structure en domaines, doit nous permettre, d'une part d'avoir un argument supplémentaire en faveur du modèle d'anisotropie de la désaimantation d'autre part, de nous renseigner sur le caractère isotrope ou non du champ de traînage.

2. Etude de la variation angulaire du traînage de fluctuations thermiques dans le domaine de Rayleigh. Dans toute cette étude, nous envisagerons seulement un problème plan. L'échantillon étudié est assimilable a un ellipsoïde de révolution aplati suivant $\mathrm{O} y$, les champs sont appliqués dans le plan équatorial $z \mathrm{O} x$ et l'aimantation est mesurée dans ce même plan. Nous considérerons toujours les champs internes, c'est-à-dire, les champs appliqués corrigés du champ démagnétisant de forme.

2.1 ETAT DÉSAIMANTÉ ISOTROPE. - On obtient un état désaimanté isotrope quand on fait agir un champ alternatif évanescent d'amplitude maximale $\geqslant 3 H_{c}$ sur un polycristal uniaxe sans orientations préférentielles. Ce serait le cas d'un polycristal parfait de cobalt en phase hexagonale $\left(^{3}\right)$. Mais il est également possible d'obtenir un état désaimanté isotrope, à partir d'un cristal de structure cubique en faisant une désaimantation tournante. Pour cela, on fait tourner l'échantillon autour de l'axe $\mathrm{O} y$ à vitesse angulaire constante alors qu'on applique dans le plan $z \mathrm{O} x$ un champ alternatif évanescent de direction constante et d'amplitude maximale $\geqslant 3 H_{\mathrm{c}}$. Dans ce dernier cas, on moyenne l'anisotropie créée par la désaimantation. Du point de vue macroscopique, ces deux cas diffèrent seulement par la valeur de la susceptibilité différentielle obtenue dans le plan de mesure.

(2) Remarque : L'application d'un champ modifie toujours la position des parois et le volume des domaines. Il peut également modifier le nombre de parois. Mais nous avons montré [7] que la limite du domaine de Rayleigh avait une signification physique simple : c'est le champ maximum qu'on peut appliquer à une configuration en domaines sans remanier de façon sensible la répartition des vecteurs polarisation qui la caractérise. En effet, le phénomène de reptation qui est lié à de tels remaniements disparaît dans le domaine de Rayleigh.

(3) Remarque : Il s'agit là d'un cas idéal. En effet, le cobalt polycristallin en phase hexagonale est obtenu par écrouissage, ce qui entraîne l'existence de directions préférentielles. On peut améliorer la situation à l'aide de traitements thermiques convenables ; mais il apparaît alors du cobalt en phase cubique.
2.1.1 Traînage sous champ dans le domaine de Rayleigh. Calcul de l'évolution temporelle de l'aimantation en un point de la courbe de première aimantation. - Cas d'un polycristal uniaxe. - Le champ magnétisant $\mathbf{H}$ est appliqué suivant l'axe $O x$ et la mesure de l'aimantation se fait dans la direction $\mathbf{u}$ du plan $z \mathrm{O} x,(\mathbf{H}, \mathbf{u})=\alpha$ (Fig. 1). Le calculde l'ai-

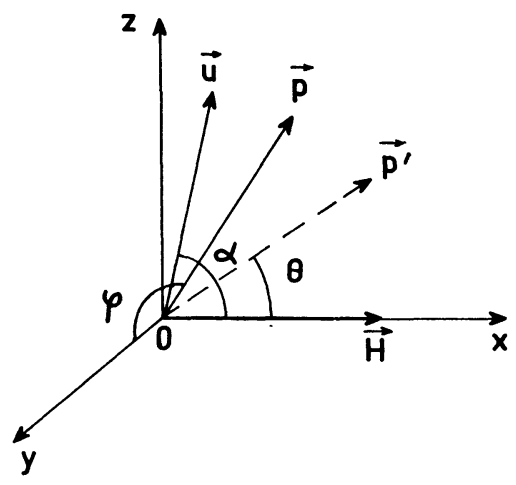

Fig. 1. - Notation relative aux angles dans le cas d'un état désaimanté isotrope. Le champ magnétisant $\mathbf{H}$, la direction de mesure $\mathbf{u}$ et le champ de désaimantation sont dans le plan $z \mathrm{O} x$.

[Definition of the angular parameters in the case of an isotropic demagnetized state. The magnetizing field $\mathbf{H}$, the direction of measurement direction $\mathrm{u}$ and the demagnetizing field $\mathfrak{H}$ lie in the $z \mathrm{O} x$ plane.]

mantation $(J)_{\text {a }}$ est en tout point analogue à celui que nous avons développé dans [6]. Il consiste à remarquer que dans certaines conditions discutées dans ce mémoire, une structure en domaines élémentaires est équivalente à une répartition de vecteurs de polarisation, chacun d'eux étant affecté à une paroi. Nous avons montré par ailleurs [7] que le traînage de fluctuations thermiques garde un sens au niveau d'une seule paroi de Bloch. Pour celà, nous l'avons mis en évidence sur un cadre monocristallin de Fe-Si dont la structure en domaine comporte une seule paroi à $180^{\circ}$ mobile. Il est donc légitime d'écrire l'aimantation suivant une direction de polarisation en tenant compte du traînage. On la projette suivant la direction de mesure et on somme sur toutes les directions de polarisation compte-tenu de leur répartition. Pour mener le calcul jusqu'au bout, il faut se donner la répartition angulaire du champ de traînage ; nous le supposerons isotrope. Nous avons fait ce calcul dans l'annexe 1 . On obtient :

$$
(J)_{\mathbf{u}}=\cos \alpha\left\{\frac{a}{3} H+\frac{b}{4} H^{2}+\frac{2 b H}{3} h_{\mathrm{t}}\right\}
$$

où $a$ et $b$ sont les constantes de la loi de Rayleigh suivant une direction de polarisation; soit pour la partie de l'aimantation qui dépend du temps :

$$
\begin{aligned}
\left(J_{\mathbf{w}}\right)_{t} & =\frac{2 b H}{3} \cos \alpha h_{\mathrm{t}} \\
& =S_{\mathrm{i}} \cos \alpha S(Q+\log t) .
\end{aligned}
$$


Entre deux instants $t_{1}$ et $t_{2}$, l'aimantation varie de la quantité :

$$
\left(\Delta J_{w}\right)_{t_{1}, t_{2}}=S_{\mathrm{i}} \cos \alpha S \log \frac{t_{2}}{t_{1}} .
$$

Il est à remarquer qu'un champ pluridirectionnel agit sur une répartition en domaines donnée plus efficacement qu'un champ unidirectionnel de même amplitude. La susceptibilité différentielle irréversible vaut en effet $\frac{2 b H}{3}$ au lieu de $\frac{2 b H}{4} . S_{\mathrm{i}}$ dépend de la répartition spatiale du champ de mesure.

Cas d'un polycristal cubique. - Le résultat découle du calcul de l'aimantation dans le cas d'un état désaimanté anisotrope, nous les donnons dans l'annexe 1 .

Le résultat est analogue au précédent, seule change la valeur de $S_{\mathrm{i}}$. On a :

$$
S_{\mathrm{i}}=2 H\left\{b_{1}^{\prime} Q_{1 \mathrm{r}}^{t}+b_{4}^{\prime} Q_{4 \mathrm{r}}^{t}+\frac{b^{\prime}}{3}\right\} \text {. }
$$

Les significations des divers termes du crochet sont précisées dans l'annexe 1 et dans le mémoire [5].

2.1.2 Détermination du champ de traînage dans le domaine de Rayleigh. - Les expériences de traînage sous champ permettent de déterminer la quantité $S_{\mathrm{i}} S$ et sa variation angulaire. Nous allons maintenant décrire une méthode permettant d'avoir accès à $h_{\mathbf{t}}$. Nous nous bornerons au cas d'un polycristal parfait de structure uniaxiale. Partant de l'état désaimanté de référence, on applique à l'échantillon le programme de champ décrit par la figure 3. On mesure les aimantations $J_{1}, J_{2}, J_{3}$, obtenues aux instants $t ; t+t^{\prime}=2 t$; et $t+t^{\prime}+t^{\prime \prime}=3 t$, la fin de la désaimantation étant prise comme origine des temps $\left({ }^{4}\right)$. H et h sont dans le plan $z \mathrm{O} x$. H est suivant

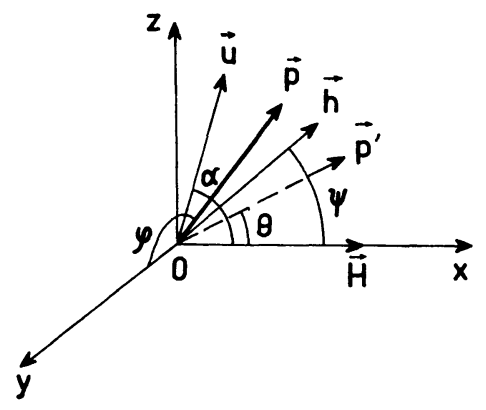

FIG. 2. - Notation relative aux angles dans le cas d'un état désaimanté isotrope. Le champ magnétisant $\mathbf{H}$, le champ additionnel $\mathbf{h}$, la direction de mesure $\mathbf{u}$ et le champ de désaimantation sont dans le plan $z \mathrm{O} x$.

[Definition of the angular parameters in the case of an isotropic demagnetized state. The magnetizing field $\mathbf{H}$, the additional field $h$, the direction of measurement $\mathbf{u}$ and the demagnetizing field $\mathscr{H}$ lie in the $z \mathrm{O} x$ plane.]

(4) Remarque : Ceci dans le but de rendre reproductible un éventuel traînage de diffusion.

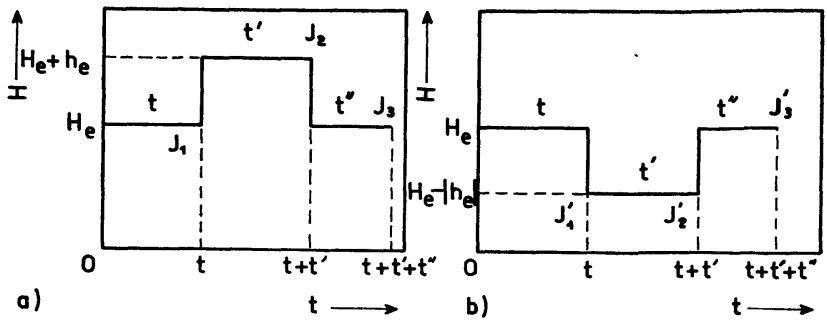

FIG. 3. - Schéma de l'application des champs $\mathbf{H}$ et $\mathbf{h}$ pendant les temps $t$ et $t^{\prime}$.a) les projections $H_{\mathrm{e}}$ et $h_{\mathrm{e}}$ sont de même signe;

b) $H_{\mathrm{e}}$ et $h_{\mathrm{e}}$ sont de signe contraire.

[Time schedule of application of fields $\mathbf{H}$ and $\mathbf{h} . a$ ) The projections $H_{\mathrm{e}}$ and $h_{\mathrm{e}}$ have the same sign. b) $H_{\mathrm{e}}$ and $h_{\mathrm{e}}$ have opposite signs.]

$\mathrm{O} x,(\mathrm{O} x, \mathbf{h})=\psi$ et l'aimantation est mesurée dans la direction $\mathbf{u}$ de ce plan : $(\mathrm{O} x, \mathbf{u})=\alpha$ (Fig. 2).

Nous allons nous intéresser à la variation, en fonction de l'amplitude du champ $\mathbf{h}$, de quantités du type $\left(\Delta J_{\psi, \alpha}\right)^{1}$ et $\left(\Delta J_{\psi, \alpha}\right)^{2} . \psi$ se rapporte à la direction du champ h et $\alpha$ à la direction de mesure de l'aimantation. Les indices 1 et 2 ont trait respectivement aux quantités $\left(J_{3}-J_{1}\right)$ et $\left(J_{3}-J_{2}\right)$. Nous mesurerons les quantités :

$$
\left\{\begin{array}{l}
\left(\Delta J_{0,0}\right)^{2}=f_{1}(h) \\
\left(\Delta J_{\pi / 2,0}\right)^{2}=f_{2}(h) \\
\left(\Delta J_{0,0}\right)^{1}=f_{3}(h) .
\end{array}\right.
$$

Le calcul (cf. annexe 1) donne les expressions de ces trois quantités. On a :

$$
\left\{\begin{array}{l}
\left(\Delta J_{0,0}\right)^{2}=-\frac{1}{3}\left(a+2 b h_{t}\right) h-\frac{b}{4} \frac{h^{2}}{2} \\
\left(\Delta J_{\pi / 2,0}\right)^{2}=\frac{2}{\pi}\left(-\frac{2}{3} b h_{t}\right) h \\
\left(\Delta J_{0,0}\right)^{1}=\frac{b H}{2} h+\frac{b h^{2}}{8} .
\end{array}\right.
$$

De $\left(\Delta J_{\pi / 2,0}\right)^{2}=f_{2}(h)$, on tire le produit $b h_{\mathrm{t}}$; la connaissance de $\left(\Delta J_{0,0}\right)^{1}=f_{3}(h)$ donne $b$. On a donc $h_{\mathrm{t}} \cdot\left(\Delta J_{0,0}\right)^{2}=f_{1}(h)$ permet d'une part d'avoir accès à la quantité $a$, d'autre part de vérifier le modèle car on peut en tirer la quantité $b$. La connaissance des coefficients $a$ et $b$ de la loi de Rayleigh élémentaire ainsi que celle du champ de traînage $h_{\mathrm{t}}$ permet de calculer par exemple $\left(\Delta J_{\psi, 0}\right)^{2}$ en fonction de $\psi$. Cette quantité étant mesurable, il est possible de comparer son évolution en fonction de $\psi$ à celle que donne le calcul. S'il n'y a pas d'écart significatif, c'est que l'hypothèse du champ de traînage isotrope est correcte, car nous avons vérifié par ailleurs le modèle.

2.2 ETAT DÉSAIMANTÉ ANISOTROPE. - Nous envisagerons seulement le cas d'un polycristal de structure cubique ayant comme le fer une grande anisotropie et ses axes de facile aimantation suivant les axes qua- 
ternaires du cristal $\left(K_{1}>0\right)$, désaimanté dans un champ alternatif évanescent. L'état initial anisotrope est obtenu en appliquant une désaimantation tournante suivie d'une désaimantation uniaxiale de même amplitude $\mathscr{H}_{\mathrm{m}} \geqslant 3 H_{\mathrm{c}}$.

2.2.1 Traînage sous champ dans le domaine de Rayleigh. Calcul de l'évolution temporelle de l'aimantation en un point de la courbe de première aimantation. - Le champ de désaimantation $\mathscr{H}$ est toujours appliqué suivant $\mathrm{O} x$, le champ magnétisant $\mathbf{H}$ fait avec l'axe Ox l'angle $\gamma$, on mesure l'aimantation soit suivant $H$, soit suivant une direction $\mathbf{u}$ telle que $(\mathrm{O} x, \mathbf{u})=\alpha$ (Fig. 4).

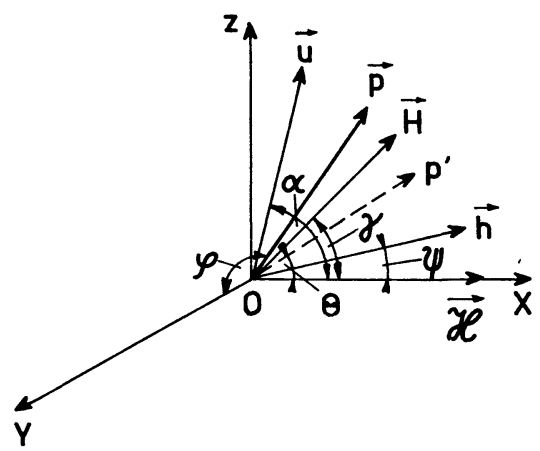

Fig. 4. - Notation relative aux angles dans le cas d'un état désaimanté anisotrope. Le champ magnétisant $\mathbf{H}$, le champ additionnel $h$, le champ de désaimantation $\boldsymbol{H}$ et la direction de mesure u sont dans le plan $z \mathrm{O} x$.

[Definition of the angular parameters in the case of an anisotropic demagnetized state. The magnetizing field $\mathbf{H}$, the additional field $h$, the demagnetizing field $\mathscr{H}$ and the measurement direction $\mathbf{u}$ lie in the $z \mathrm{O} x$ plane.]

Le calcul de la variation d'aimantation $\left(\Delta J_{\mathbf{H}}\right)_{t}$ est donné dans l'annexe 1 . On obtient :

$$
\left(\Delta J_{\mathbf{H}}\right)_{t}=2 H h_{\mathbf{t}}\left\{\frac{b^{\prime}}{3}+b_{1}^{\prime} Q_{1, \mathbf{r}}^{\gamma}+b_{2}^{\prime} Q_{4 \mathrm{r}}^{\gamma}\right\}
$$

Les quantités $b^{\prime}, b_{1}^{\prime}, b_{2}^{\prime}$ sont les constantes de Rayleigh microscopiques des divers types de parois considérées dans le modèle; elles sont accessibles à partir de mesures de susceptibilité réalisées de façon à éliminer l'influence du traînage. Les quantités $Q$ sont calculables à partir du modèle.

La variation d'aimantation sous champ entre les instants $t_{1}$ et $t_{2}$ s'écrit en exprimant $h_{\mathrm{t}}$ :

$$
\begin{aligned}
\left(\Delta J_{\mathbf{H}}\right)_{t_{1}, t_{2}} & =2 H T(\gamma) S \log \frac{t_{2}}{t_{1}} \\
& =S_{\mathrm{i}} S \log \frac{t_{2}}{t_{1}}
\end{aligned}
$$

avec

$$
S_{\mathrm{i}}=2 H T(\gamma)=2 H\left\{\frac{b^{\prime}}{3}+b_{1}^{\prime} Q_{1 \mathrm{r}}^{\gamma}+b_{2}^{\prime} Q_{4 \mathrm{r}}^{\gamma}\right\}
$$

La remarque que nous avions faite en 2.1.1, reste valable ici. $S_{\mathrm{i}}$ est plus grande que celle qu'on obtiendrait en partant du même état désaimanté et en la mesurant avec un champ de symétrie uniaxiale.

On mesure la quantité :

$$
\frac{\left(\Delta J_{H}\right)_{t_{1}, t_{2}}}{\log \frac{t_{2}}{t_{1}}}=f(\gamma)
$$

et on compare le résultat obtenu à celui qu'on calcule à partir de $T(\gamma)$.

3. Vérification expérimentale. - L'analyse que nous avons faite de la variation directionnelle du traînage de fluctuations suggère une vérification expérimentale en deux temps. Nous vérifierons d'abord que le modèle d'anisotropie de la désaimantation permet de prévoir l'essentiel des phénomènes observés. Pour cela, une mesure de traînage sous champ dans le domaine de Rayleigh est la plus simple à mettre en œuvre. Elle doit nous permettre de fixer l'ordre de grandeur de l'anisotropie du 'champ de traînage. S'il s'avère que celui-ci est presque isotrope, nous serons conduits, dans un deuxième temps, à faire une étude plus détaillée de cette anisotropie à partir de la détermination du champ de traînage. Nous nous sommes bornés à la première partie de la vérification.

3.1 MÉThOde DE MESURE. - Nous avons utilisé l'échantillon nous ayant servi à l'étude de l'anisotropie de la désaimantation. Il s'agit d'un disque mince en acier : $\varnothing: 60 \mathrm{~mm}, e=1,2 \mathrm{~mm}$. Nous l'avons assimilé à un ellipsoïde de révolution aplati dont le plan équatorial $z \mathrm{O} x$ et confondu avec le plan du disque. Nous avons adopté comme coefficient de champ démagnétisant celui de l'ellipsoïde soit $N=0,20$. Le choix du matériau n'est pas critique. La composition en poids de cet acier était : $\mathrm{Fe} 93,4 \% ; \mathrm{Ni} \mathrm{4,3 \%}$; $\mathrm{Cr} 1,5 \%$; Mo $0,5 \% ; \mathrm{C} 0,3 \%$. Il a été recuit pendant $1 \mathrm{~h}$ à $650^{\circ}$ en atmosphère d'hydrogène et refroidi en $24 \mathrm{~h}$. C'est un polycristal de structure cubique, sans orientation préférentielle notable, de comportement analogue à celui du fer. Son champ coercitif est 14,7 Oe dans le plan $z \mathrm{O} x$. Un ensemble de bobines permet d'appliquer le champ magnétisant $\mathbf{H}$, le champ alternatif de désaimantation $H$ dans une direction quelconque de $z \mathrm{O} x$. Nous avons mesuré l'aimantation à l'aide d'un fluxmètre électronique $[8,9]$. Après avoir désaimanté l'échantillon, on applique le champ magnétisant et on enregistre à champ constant la variation d'aimantation. En pratique, pour éviter de saturer le fluxmètre lors de l'application du champ, l'enregistrement ne débute que 2 à 3 secondes après son application; il se poursuit pendant une centaine de secondes. Dans ces mesures, le champ appliqué à l'échantillon vaut 1,22 Oe. Dans ces conditions, les lois de Rayleigh sont suivies à mieux que $10^{-3}$ près.

3.2 ETAT DÉSAIMANTÉ ISOTROPE. - Comme il s'agit d'un échantillon de structure cubique, l'état de réfé- 
rence est obtenu en faisant une désaimantation tournante d'amplitude $>3 H_{\mathrm{c}}$. La figure 5 donne un exemple d'enregistrement obtenu. Les points de cette

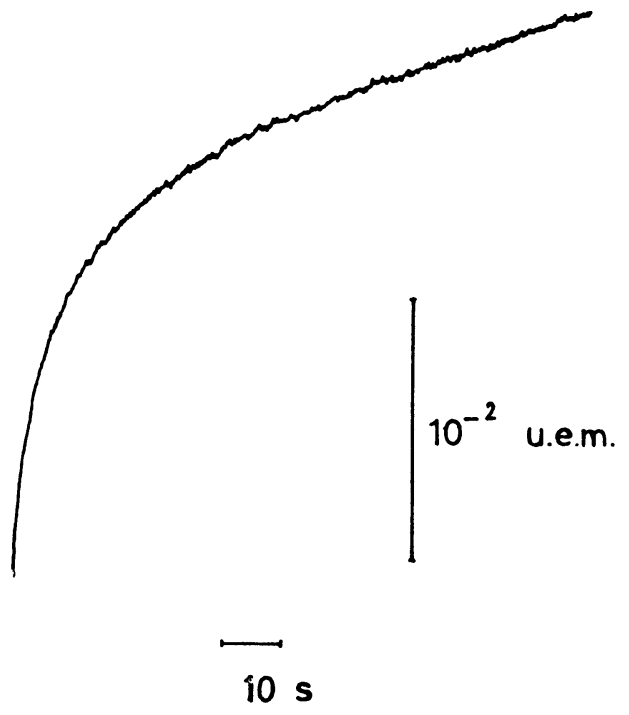

Fig. 5. - Exemple d'enregistrement de $(\Delta J)_{t_{1}, t_{2}}$. La variation de flux correspondante est de l'ordre de 20 maxwells; la dérive du fluxmètre n'excède pas $\pm 0,15$ maxwell durant la mesure.

[A typical recording of $(\Delta J)_{t_{1}, t_{2}}$. The corresponding change of flux is about 20 maxwells; the drift of the fluxmeter does not exceed \pm 0.15 maxwell during the measurement.]

courbe, lorsqu'on les porte en fonction de $\log t$, se placent sur une droite de manière très satisfaisante (Fig. 6). La pente de la droite donne la valeur expérimentale de la quantité $S_{\mathrm{i}} S$.

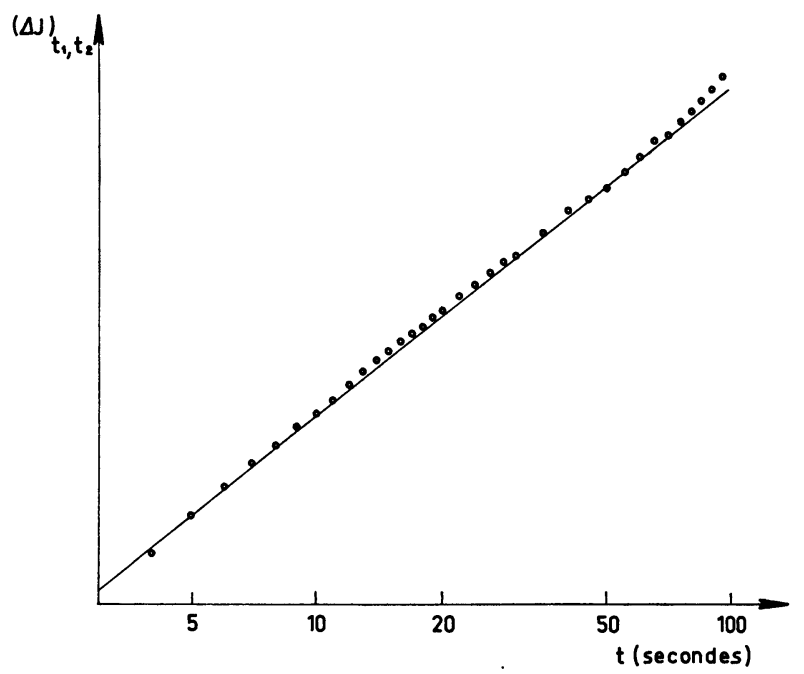

FIG. 6. - Aspect de $(\Delta J)_{t_{1}, t_{2}}$ en fonction de $\log t$. $\left[(\Delta J)_{t_{1}, t_{2}}\right.$ plotted versus $\left.\log t.\right]$

On remarque sur l'enregistrement de la figure 5 un bruit. Sa valeur crête à crête est nettement supérieure à celle de l'appareillage de mesure, ce qui nous incite à attribuer les irrégularités de la courbe à l'activité magnétique microscopique due au traînage de fluctuations. L'ordre de grandeur du bruit correspond à des retournements de volumes de matière aimantée de $2 \times 10^{-7} \mathrm{~cm}^{3}$ environ. Cette valeur est compatible avec les déterminations des volumes de sauts ou de groupes de sauts Barkhausen dont l'amplitude maximale dépend de la substance mais peut atteindre quelques $10^{-7} \mathrm{~cm}^{3}$ dans des matériaux polycristallins à base de fer ou de nickel $[10,11]$. Il est intéressant de remarquer que nos enregistrements de traînage révèlent à la fois le phénomène élémentaire et sa moyenne à notre échelle qui se traduit par la loi en log $t$.

Nous avons vérifié qu'à partir de cet état de référence, le traînage dans le plan $z \mathrm{O} x$ était isotrope. Pour cela, nous l'avons mesuré dans les mêmes conditions dans deux directions perpendiculaires de ce plan.

Nous avons obtenu pour la quantité $10^{3} S_{\mathrm{i}} S$ : $3,67 \pm 0,08$ et 3,68 $\pm 0,08$, ces valeurs étant exprimées en u. e. $\mathrm{m}$. Le calcul fait à partir du modèle donne pour cette même quantité : 3,71 u. e. m. (Fig. 7).

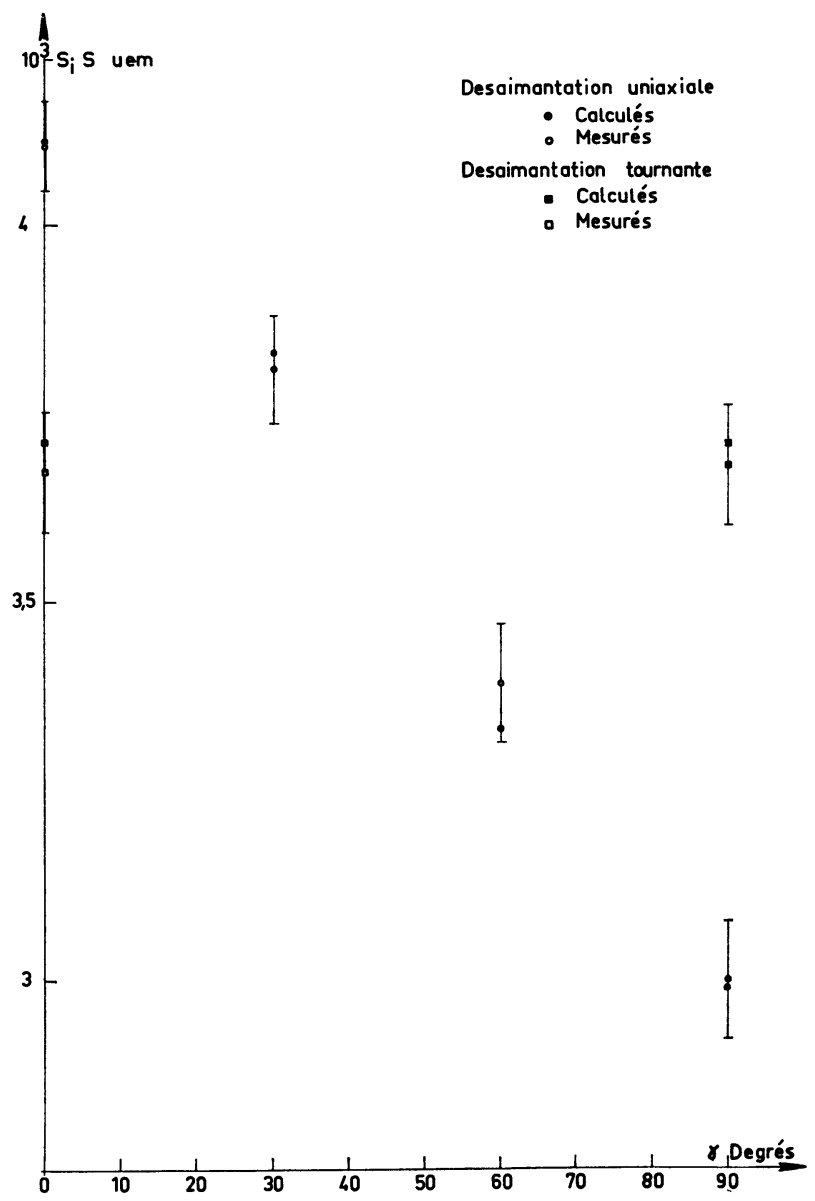

Fig. 7. - Valeurs de $S_{\mathrm{i}} S$ mesurées et calculées en fonction de l'angle $\gamma=(\Re,, \mathbf{H})$, dans le cas où $\alpha=\gamma$. On a également porté sur le graphique les valeurs calculées et mesurées de $S_{\mathrm{i}} S$ dans le cas d'une désaimantation tournante pour deux directions perpendiculaires du plan $z \mathrm{O} x$.

[Measured and calculated values of $S_{\mathrm{i}} S$ as functions of the angle $\gamma=(\mathfrak{H}, \mathbf{H})$ in the case $\alpha=\gamma$. The diagram also indicates the measured and calculated values of $S_{\mathrm{i}} S$ after a rotating demagnetization for two perpendicular directions in the $z \mathrm{O} x$ plane.] 
De ces mesures, on détuit la valeur de la constante $S$ connaissant la valeur de la susceptibilité différentielle irréversible $S_{\mathbf{i}}$. On trouve $\mathrm{S}=15,0 \mathrm{mOe}$, soit $\# H_{\mathrm{c}} / 1000$.

3.3 ETAT DÉSAIMANTÉ ANISOTROPE. - L'état de référence est obtenu en faisant suivre une désaimantation tournante par une désaimantation uniaxiale de même amplitude $\left(H_{\mathrm{m}}>3 H_{\mathrm{c}}\right)$. $\mathcal{H}$ est toujours dirigé suivant $\mathrm{O} x ; \mathbf{H}$ fait avec $\mathrm{O} x$ un angle $\gamma$. L'état désaimanté ainsi obtenu est anisotrope et la quantité $S_{\mathrm{i}} S$ est une fonction décroissante de $\gamma$, quand celui-ci varie de 0 à $\pi / 2$. Nous avons porté sur la figure $7, S_{\mathrm{i}} S$ en fonction de $\gamma$. Ces valeurs correspondent à des moyennes sur au moins huit mesures. Nous avons porté sur le même graphique les points calculés dans le cadre du modèle d'anisotropie de la désaimantation en supposant le champ de traînage isotrope. Les barres d'erreur indiquent la dispersion des résultats expérimentaux à partir de la valeur moyenne en prenant en considération l'écart quadratique moyen. La préci sion relative de ces mesures est de l'ordre de $\pm 2 \%$. Il est cependant bon de noter que les flux mis en jeu dans les mesures des $\Delta J$ (Fig. 5) sont de l'ordre de 20 maxwells et que, la dérive du fluxmètre durant toute la mesure n'excède pas $\pm 0,15$ maxwell.

Dans l'ensemble, l'accord est satisfaisant. On remarque cependant que les points expérimentaux sont au-dessous des points calculés pour $\gamma=0^{\circ}$ et $30^{\circ}$ et au-dessus pour $\gamma=60^{\circ}$ et $90^{\circ}$. En supposant que cet écart soit significatif, cela voudrait dire que le champ aléatoire de fluctuations thermiques présenterait une légère anisotropie défavorisant les directions où la densité de polarisation est maximale.

4. Conclusion. - Cette étude montre et l'expérience confirme, que l'anisotropie du traînage de fluctuations thermiques est une conséquence de l'anisotropie de la répartition des domaines élémentaires. L'essentiel de la variation angulaire de celui-ci est due au fait que la susceptibilité différentielle irréversible est une fonction de la direction dans laquelle on fait la mesure.

En toute rigueur, le champ de traînage dépend également de la configuration en domaines mais cette dépendance ne se traduira que par un effet de second ordre sur la variation angulaire du traînage.

Dans le cas où l'état anisotrope étudié a été créé par une désaimantation par un champ alternatif évanescent, la variation angulaire du traînage que l'on observe s'interprète en première approximation dans le cadre du modèle d'anisotropie de la désaimantation en supposant le champ de traînage isotrope. Cependant, un examen plus serré des résultats expérimentaux, laisse prévoir une légère anisotropie du champ de traînage. Seule une étude directionnelle fine de $h_{\mathrm{t}}$ permettra de trancher avec certitude.

Annexe I. - Trainage Sous Champ dans le domaine DE RAYLEIGH : CALCUL DE L'ÉVOLUTION TEMPORELLE DE L'AIMANTATION EN UN POINT DE LA COURBE DE PREMIÈRE AIMANTATION. DÉTERMINATION DU CHAMP DE TRAINAGE. - Dans ce qui suit, nous nous reférerons aux deux articles [5, 6]. Nous utiliserons les mêmes notations et les mêmes procédés de calcul ; le lecteur s'y reportera utilement.

1. Etat désaimanté isotrope. - 1.1 Trainage sous CHAMP. - 1.1.1 Cas du polycristal parfait de structure uniaxiale. (cf [6] p. 198). - L'aimantation suivant une direction de polarisation s'écrit :

$$
\delta J=a H_{\mathrm{e}}+b H_{\mathrm{e}}^{2}+2 b H_{\mathrm{e}} h_{\mathrm{t}} .
$$

$a$ et $b$ sont les constantes de la loi de Rayleigh relative à une paroi à $180^{\circ}$. Il est en effet légitime de considérer seulement ce type de paroi dans un polycristal uniaxe à forte anisotropie (cobalt). $H_{\mathrm{e}}$ est la projection de $\mathbf{H}$ sur la direction de polarisation considérée. Nous supposons le champ de traînage isotrope ; il aura la même valeur $h_{\mathrm{t}}$ suivant toutes les directions de polarisation.

La figure 1 précise la géométrie du problème. Pour calculer l'aimantation, on exprime $\delta J$ en fonction des variables introduites et on somme sur toutes les directions de polarisation en remarquant qu'elles sont toutes équiprobables $\left({ }^{1}\right)$. On obtient pour $\left(J_{u}\right)$ les expressions :

$$
\left(J_{\mathbf{u}}\right)=\frac{a H}{\pi} \int_{-\pi / 2}^{+\pi / 2} \cos \theta \cos (\alpha-\theta) \mathrm{d} \theta \int_{0}^{\pi / 2} \sin ^{3} \varphi \mathrm{d} \varphi
$$

$$
\begin{gathered}
+\frac{b H^{2}}{\pi} \int_{-\pi / 2}^{+\pi / 2} \cos ^{2} \theta \cos (\alpha-\theta) \mathrm{d} \theta \int_{0}^{\pi / 2} \sin ^{4} \varphi \mathrm{d} \varphi \\
+\frac{2 b H h_{\mathrm{t}}}{\pi} \int_{-\pi / 2}^{+\pi / 2} \cos \theta \cos (\alpha-\theta) \mathrm{d} \theta \int_{0}^{\pi / 2} \sin ^{3} \varphi \mathrm{d} \varphi \\
\left(J_{\mathrm{u}}\right)=\cos \alpha\left\{\frac{a}{3} H+\frac{b}{4} H^{2}+\frac{2 b H}{3} h_{\mathrm{t}}\right\} .
\end{gathered}
$$

Soit pour $\left(J_{u}\right)_{t}$ :

$$
\left(J_{\mathbf{u}}\right)_{t}=\cos \alpha \frac{2 b H}{3} h_{\mathrm{t}}=\cos \alpha S_{\mathrm{i}} h_{\mathrm{t}}
$$

(1) Remarque : C'est la répartition isotrope des polarisations qui introduit le facteur $1 / \pi$ devant les intégrales. 
1.1.2 Cas du polycristal par fait de structure cubique. - Nous le traiterons dans 2.2 après le cas du traînage sous champ d'un état désaimanté anisotrope ; car il en découle simplement.

1.2 DÉTERmination du ChAmp DE TRAINAge DANS Le domaine DE RAYleigh (cf. (5), p. 178 à 182 ). Nous envisagerons uniquement le cas d'un polycristal parfait de structure uniaxiale.

Partant de l'état désaimanté de référence, on applique le champ $\mathbf{H}$ pendant un temps $t$, le champ $\mathbf{H}$ et $\mathbf{h}$ pendant un temps $t^{\prime}$ et à l'instant $t+t^{\prime}$, on supprime le champ additionnel $\mathbf{h}$, La fin de la désaimantation définit l'origine des temps et la direction de $\mathbf{h}$ est quelconque dans le plan $z \mathrm{O} x$ (Fig. 2). Suivant la direction de polarisation considérée, on peut avoir deux situations : les projections $H_{\mathrm{e}}$ et $h_{\mathrm{e}}$ de $\mathbf{H}$ et $\mathbf{h}$ sont de même signe ou de signe contraire (Fig. 3). L'étude de l'influence du traînage de fluctuations thermiques nous permet d'écrire, dans le cadre de la théorie de Néel, les expressions $J_{1}, J_{2}, J_{3} ; J_{1}^{\prime}, J_{2}^{\prime}, J_{3}^{\prime}$ (cf. [5] p. 179). A l'aide de ces expressions, on peut évaluer dans les deux cas envisagés, les variations d'aimantation successives dues à l'application et à la suppression de $\mathbf{h}:\left(J_{2}-J_{1}\right),\left(J_{1}^{\prime}-J_{2}^{\prime}\right)$ et $\left(J_{3}-J_{2}\right),\left(J_{3}^{\prime}-J_{2}^{\prime}\right)$ ainsi que la variation d'aimantation totale : $\left(J_{3}-J_{1}\right),\left(J_{3}^{\prime}-J_{1}^{\prime}\right)$.

Moyennant certaines hypothèses qui sont discutées dans [5] on peut simplifier les expressions de ces variations d'aimantation dans le cas particuier où $t=t^{\prime}=t^{\prime \prime}$.

Soit $(\Delta J)^{1}$ et $(\Delta J)^{2}$, les variations d'aimantation de l'échantillon entre les instants $t$ et $t+t^{\prime}+t^{\prime \prime}=3 t$ d'une part et les instants $t+t^{\prime}=2 t$ et $t+t^{\prime}+t^{\prime \prime}=3 t$ d'autre part. Les expressions de l'aimantation suivant une direction de polarisation sont données dans le premier cas par $\left(J_{3}-J_{1}\right),\left(J_{3}^{\prime}-J_{1}^{\prime}\right)$ et par $\left(J_{3}-J_{2}\right)$, $\left(J_{3}^{\prime}-J_{2}^{\prime}\right)$ dans le second. Nous aurons chaque fois deux sommations à faire l'une portant sur la différence simple quand $H_{\mathrm{e}}$ et $h_{\mathrm{e}}$ sont de même signe, l'autre portant sur la différence primée dans le cas où $H_{\mathrm{e}}$ et $h_{\mathrm{e}}$ sont de signe contraire. On a suivant une direction de polarisation :

$$
\begin{aligned}
& \left\{\begin{array}{l}
J_{2}-J_{1}=a h_{\mathrm{e}}+2 b H_{\mathrm{e}} h_{\mathrm{e}}+b h_{\mathrm{e}}^{2}+2 b h_{\mathrm{e}} h_{\mathrm{t}} \\
J_{3}-J_{2}=-a h_{\mathrm{e}}-\frac{b}{2} h_{\mathrm{e}}^{2}-2 b h_{\mathrm{e}} h_{\mathrm{t}} \\
J_{3}-J_{1}=2 b H_{\mathrm{e}} h_{\mathrm{e}}+\frac{b}{2} h_{\mathrm{e}}^{2}
\end{array}\right. \\
& \left\{\begin{array}{l}
J_{2}^{\prime}-J_{1}^{\prime}=-a\left|h_{\mathrm{e}}\right|-\frac{b}{2} h_{\mathrm{e}}^{2}-2 b\left|h_{\mathrm{e}}\right| h_{\mathrm{t}} \\
J_{3}^{\prime}-J_{2}^{\prime}=a\left|h_{\mathrm{e}}\right|+\frac{b}{2} h_{\mathrm{e}}^{2}+2 b\left|h_{\mathrm{e}}\right| h_{\mathrm{t}} \\
J_{3}^{\prime}-J_{1}^{\prime}=0
\end{array}\right.
\end{aligned}
$$

où $a$ et $b$ sont les constantes élémentaires de la loi de Rayleigh relatives à la paroi considérée et $h_{\mathrm{t}}$ le champ de traînage supposé isotrope.

La géométrie du problème est donnée par la figure 2. Nous adopterons pour la variation d'aimantation, une notation a deux indices, le premier $\psi$ ayant trait à la direction de $\mathbf{h}$, le second $\alpha$ caractérisant la direction de mesure u. Evaluons $\left(\Delta J_{\psi, \alpha}\right)^{2}$, pour cela, sommons sur toutes les directions de polarisation en remarquant qu'elles sont équiprobables. On obtient :

$$
\begin{aligned}
\left(\Delta J_{\psi, \alpha}\right)^{2}=-h a \frac{1}{\pi} \int_{-\pi / 2}^{+\pi / 2} \cos (\psi-\theta) \cos (\alpha-\theta) \mathrm{d} \theta \int_{0}^{\pi / 2} \sin ^{3} \varphi \mathrm{d} \varphi & -\frac{h^{2}}{2} b \frac{1}{\pi}\left[\int_{-\pi / 2}^{\pi / 2} \cos ^{2}(\psi-\theta) \cos (\alpha-\theta) \mathrm{d} \theta\right]_{0}^{*} \int^{\pi / 2} \sin ^{4} \varphi \mathrm{d} \varphi \\
& -2 h h_{\mathrm{t}} b \frac{1}{\pi}\left[\int_{-\pi / 2}^{+\pi / 2} \cos (\psi-\theta) \cos (\alpha-\theta) \mathrm{d} \theta\right]_{0}^{* *} \int^{\pi / 2} \sin ^{3} \varphi \mathrm{d} \varphi
\end{aligned}
$$

avec la convention ci-dessous.

Pour les polarisations où $H_{\mathrm{e}}$ et $h_{\mathrm{e}}$ sont de même signe, on prend le signe + pour les crochets []$^{*}$ et []$^{* *}$. Dans le cas contraire, on prend le signe pour [ ]*, alors que dans [ $]^{* *}$ le signe de $h_{\mathrm{e}}$ est celui de $H_{\mathrm{e}}$, l'élément d'intégration étant affecté du signe -.

Envisageons les deux cas particuliers suivants: $\psi=0, \alpha=0$ qui correspond à la variation d'aiman- 
tation longitudinale et $\psi=\pi / 2, \alpha=0$ qui est la variation d'aimantation longitudinale correspondant à l'application d'un champ additionnel transversal. Le résultat du calcul s'écrit :

$$
\begin{aligned}
& \left(\Delta J_{0,0}\right)^{2}=-\frac{1}{3}\left(a+2 b h_{t}\right) h-\frac{b}{4} \frac{h^{2}}{2} \\
& \left(\Delta J_{\pi / 2,0}\right)^{2}=\frac{2}{\pi}\left(-\frac{2}{3} b h_{t}\right) h .
\end{aligned}
$$

On calculerait de la même façon $\left(\Delta J_{\psi, \alpha}\right)^{1}$ à partir des expressions de $\left(J_{3}-J_{1}\right),\left(J_{3}^{\prime}-J_{1}^{\prime}\right)$. Ici le calcul se simplifie car $\left(J_{3}^{\prime}-J_{1}^{\prime}\right)=0$. On trouve pour la variation d'aimantation longitudinale, c'est-à-dire pour $\psi=0, \alpha=0$ :

$$
\left(\Delta J_{0,0}\right)^{1}=h \frac{b H}{2}+\frac{b h^{2}}{8}
$$

soit

$$
S_{\mathrm{i}(00)}=\frac{b H}{2}
$$

2. Etat désaimanté anisotrope. - 2.1 Trainage SOUS CHAMP. (cf. (5) p. 174 à 178). - Dans le cas que nous avons envisagé, nous sommes amenés à considérer trois types de parois. Dans la partie du cristal qui s'oriente : phase I, les polarisations du type 1 et 4 interviennent. Elles correspondent respectivement aux parois à $180^{\circ}$ et $90^{\circ}$ formées en associant deux à deux les directions de facile aimantation situées le plus favorablement par rapport à la direction de désaimantation. Il faut également tenir compte de la fraction non orientable du cristal (phase II) où, par hypothèse, la répartition des polarisations reste isotrope quel que soit le mode de désaimantation.

Les trois types de polarisation 1, 4 et isotrope sont caractérisés par leurs constantes de Rayleigh microscopiques $a_{1}^{\prime}$ et $b_{1}^{\prime}, a_{4}^{\prime}$ et $b_{4}^{\prime}, a^{\prime}$ et $b^{\prime}$ que nous avons pu déterminer moyennant certaines hypothèses [6].

A chaque type de polarisation est associée une répartition qui lui est propre soit respectivement pour les parois 1, 4 de la phase I et pour la paroi moyenne de la phase II : $P_{1}^{\prime}(\varphi, \theta), \mathrm{P}_{4}^{\prime}(\varphi, \theta)$ et $1 / \pi$. La figure 4 précise la géométrie du problème. En supposant le champ de traînage isotrope et en nous bornant au terme qui varie en fonction du temps, on obtient :

$$
\begin{aligned}
(\Delta J)_{\mathbf{u}}=2 b_{1}^{\prime} H h_{\mathrm{t}} \int_{-\pi / 2+\gamma}^{\pi / 2+\gamma} \cos (\gamma-\theta) \cos (\alpha-\theta) \mathrm{d} \theta \int_{0}^{\pi / 2} P_{1}^{\prime}(\varphi, \theta) \sin ^{3} \varphi \mathrm{d} \varphi \\
+2 b_{2}^{\prime} H h_{\mathrm{t}} \int_{-\pi / 2+\gamma}^{\pi / 2+\gamma} \cos (\gamma-\theta) \cos (\alpha-\theta) \mathrm{d} \theta \int_{0}^{\pi / 2} P_{4}^{\prime}(\varphi, \theta) \sin ^{3} \varphi \mathrm{d} \varphi \\
+2 b^{\prime} H h_{t} \int_{-\pi / 2+\gamma}^{\pi / 2+\gamma} \cos (\gamma-\theta) \cos (\alpha-\theta) \mathrm{d} \theta \int_{0}^{\pi / 2} \frac{1}{\pi} \sin ^{3} \varphi \mathrm{d} \varphi
\end{aligned}
$$

Si la mesure se fait suivant $\mathbf{H}$, il suffit de faire $\alpha=\gamma$, on obtient alors :

$$
(\Delta J)_{\mathbf{H}}=2 H h_{\mathrm{t}}\left(b_{1}^{\prime} Q_{1 \mathrm{r}}^{\gamma}+b_{2}^{\prime \gamma} Q_{4 \mathrm{r}}^{\gamma}+\frac{b^{\prime}}{3}\right)
$$

où les $Q$ représentent les valeurs des intégrales ci-dessus.

$$
(\Delta J)_{\mathbf{H}}=H h_{\mathrm{t}} T(\gamma)
$$

en posant : $T(\gamma)$ égal à :

$$
\left(b_{1}^{\prime} Q_{1 \mathrm{r}}^{\gamma}+b_{2}^{\prime} Q_{4 \mathrm{r}}^{\gamma}+\frac{b^{\prime}}{3}\right) \text {. }
$$

Si l'état désaimanté initial est obtenu par ıne désaimantation tournante, il est alors isotrope par compen- sation dans le plan $z \mathrm{O} x$. L'expression de la variation d'aimantation s'obtient en prenant la valeur moyenne des $Q$ par rapport à $\gamma$. On a pour $\left(\Delta J_{\mathbf{H}}\right)_{t}$ :

$$
\left(\Delta J_{\mathbf{H}}\right)_{t}=2 H h_{\mathrm{t}}\left\{b_{1}^{\prime} Q_{1 \mathrm{r}}^{t}+b_{2}^{\prime} Q_{4 \mathrm{r}}^{t}+\frac{b^{\prime}}{3}\right\} .
$$

2.2 DÉTERmination DU CHAMP DE TRAINAGE DANS LE DOMAINE DE RAYleigh. - On procède de la même façon que dans le cas d'une répartition isotrope. Le calcul est ici un peu plus compliqué du fait qu'il faut considérer trois types de vecteurs polarisations ayant chacun leur répartition propre. Nous avons fait ce calcul dans [5], p. 180 à 182 ; nous ne le reprendrons pas ici, le lecteur s'y reportera utilement.

\section{Bibliographie}

[1] Ewing, J. A., Phil. trans. 176 (1885) 569.

EwING, J. A. Proc. Roy. Soc. 46 (1889) 269.

[2] NeEL, L., J. Physique Radium 11 (1950) 49.

NeEL, L., J. Physique Radium 12 (1951) 339.

[3] NeEL, L., J. Physique Radium 11 (1950) 49.

[4] Barbier, J. C., Thèse Grenoble (1953).

[5] Vergne, R., Portesen, J. L., Blažek, Z., Phys. Stat. Sol. (a), 25 (1974) 171
[6] Vergne, R., Porteseil, J. L., Blažek, Z., Revue Phys. Appl. 10 (1975) 195.

[7] Portesen, J. L., Thèse Grenoble, (1975).

[8] Portesent, J. L., Thèse $3^{e}$ Cycle, Grenoble (1969).

[9] Vergne, R., Porteseil, J. L., Revue Phys. Appl. 6 (1971) 95.

[10] Heaps, C. W., Taylor, J., Phys. Rev. 36 (1929) 937.

[11] Heaps, C. W., Bryan, A. B., Phys. Rev. 36 (1930) 326. 